\title{
Egg recovery completed with a "manually-created" negative pressure is still an option in cases of emergency or "low-cost" in vitro fertilization?
}

\author{
Theodoros Kalampokas, Abha Maheswari \\ Assisted Reproduction Unit, Aberdeen University Institute of Applied Health Sciences, Aberdeen, United Kingdom
}

\section{Abstract}

This is a report of a case of an egg recovery procedure completed with manual suction instead of the automated negative pressure suction. A 35-year-old woman who was undergoing in vitro fertilization (IVF) treatment had to undergo oocyte recovery using manual suction through a syringe instead of the automated negative pressure suction systems because of the failure of both the initial and replacement systems. The treatment cycle ended in a positive pregnancy test and a clinical pregnancy with acceptable oocyte fertilization rates and no complications during procedure. The case presented may be an implication for an alternative implementation in cases of emergency, particularly when low-cost IVF is a target. (J Turk Ger Gynecol Assoc 2015; 16: 257-8)

Keywords: IVF, oocyte retrieval, manual, suction, cost

Received: 18 April, 2015

Accepted: 13 July, 2015

Available Online Date: 02 November, 2015

\section{Introduction}

Oocyte recoveries take place with the use of sophisticated and very efficient equipment worldwide. However, sometimes during emergency conditions, such as failure of equipment of any kind, physicians are required to immediately decide regarding the future of the complete in vitro fertilization (IVF) cycle. We present a case of an oocyte recovery that was successfully completed with the use of a manually created negative pressure for oocyte aspiration because of simultaneous failure of both the main and replacement systems. It is important, primarily for less experienced operators, to know that this is an option in case of emergency conditions, particularly when IVF costs may be an issue.

\section{Case Presentation}

A 35-year-old woman, p 0, was admitted to our department with a diagnosis of unexplained primary female, male, and couple infertility with a duration of 24 months. Nothing of clinical importance could be found in the couple's medical history, except for a slightly reduced motility in the male semen analysis.

We used a fixed antagonist protocol with 225 international units of follicle-stimulating hormone/day. The last pelvic ultrasound scan performed revealed the existence of 12 good-sized follicles (>14 mm); hence, human chorionic gonadotropin triggering was decided for the following day.

On the day of oocyte recovery, we discovered that both the suction apparatus and its substitute were not working; moreover, the sooner we would have been able to have a new apparatus would have been 5-7 h later. After having an emergency meeting, we decided to go ahead with the application of a "manual" suction and a single lumen follicle aspiration needle (instead of the double lumen that we use). We applied a $20-\mathrm{mL}$ syringe, and a second doctor aspirated the follicles by creating a negative pressure using the syringe. We managed to aspirate eight oocytes from 10 suitable follicles; however, a previous ultrasonographic finding of the high left ovary was confirmed, making the procedure even more difficult.

IVF was implemented and among the eight eggs, three managed to get fertilized. A single embryo transfer of a 7c2 quality embryo took place three days later, and the other two embryos were discarded because they were not of freezing quality. The urine pregnancy test performed 15 days later proved to be positive; the patient is currently in the second trimester of an ongoing, uncomplicated pregnancy.

\section{Discussion}

The case report presented is important for two reasons: firstly, because it demonstrates a feasible and easy solution for similar instances and secondly, it may be a very cheap solution in cases where low-cost IVF is an important issue. To the best of our knowledge, this is the only case described, particularly after the implementation of the automated suction systems for oocyte recovery; a systematic review of the literature conducted revealed no other similar cases (keywords used were: "manual," "suction," "IVF," "oocyte," "aspiration," and "recovery"). 
In the past (early 1980s), follicle aspiration with manual pressure was the common procedure; nevertheless, it has been reported that follicular aspiration using a syringe suction system may damage the zona pellucida (1). This was not the case in our patient despite the lower fertilization rate that we had achieved (3/8 eggs were fertilized); however, the fact that only one good quality embryo was obtained may present a complication for the procedure.

A disadvantage of the method may be the fact that in cases of application of manual suction, we cannot apply flushing of the aspirated follicles, which is a technique used for oocyte aspiration in our center. Nevertheless, one may say that this is not an obligatory applied technique, and recent research suggests that follicles should not be flushed in cases of normal responders, as in our case (2).

In contrast, taking into consideration the fact that in many IVF centers worldwide cost may be an important issue, using a syringe to manually create the negative pressure, at least in cases of emergency, may be an attractive alternative (3). Considering that the cost of oocyte retrieval is estimated to be at approximately 220 Euro $(3,4)$, safeguarding the amount required for a pump replacement, may contribute to wider provision of IVF services under the provision of proving that manual suction leads to uncomplicated oocyte retrieval.

\section{Ethics Committee Approval: N/A.}

Informed Consent: Written informed consent was obtained from patient who participated in this case.
Peer-review: Externally peer-reviewed.

Author Contributions: Concept - T.K.; Design - T.K.; Supervision A.M.; Resource - T.K., A.M.; Materials - T.K., A.M.; Data Collection and/or Processing - T.K.; Analysis and/or Interpretation T.K., A.M.; Literature Search - T.K.; Writing - T.K.; Critical Reviews - A.M.

Conflict of Interest: No conflict of interest was declared by the authors.

Financial Disclosure: The authors declared that this study has received no financial support.

\section{References}

1. Cohen J, Avery S, Campbell S, Mason BA, Riddle A, Sharma V. Follicular aspiration using a syringe suction system may damage the zona pellucida. J In Vitro Fert Embryo Transf 1986; 3: 224-6. [CrossRef]

2. Collins J. An international survey of the health economics of IVF and ICSI. Human Reprod Update 2002; 8: 265-77. [CrossRef]

3. Levy G, Hill MJ, Ramirez CI, Correa L, Ryan ME, DeCherney AH, et al. The use of follicle flushing during oocyte retrieval in assisted reproductive technologies: a systematic review and meta-analysis. Hum Reprod 2012; 27: 2373-9. [CrossRef]

4. Bouwmans CA, Lintsen BM, Eijkemans MJ, Habbema JD, Braat DD, Hakkaart L. A detailed cost analysis of in vitro fertilization and intracytoplasmic sperm injection treatment. Fertil Steril 2008; 89: 331-41. [CrossRef] 